\title{
Influência do viés de seleção e de aferição em estimativas de tendência secular da estatura baseadas em dados da Marinha do Brasil
}

The influence of selection and measurement bias on secular trend in height estimates from Brazilian Navy data

\section{Gilberto Kac}

Departamento de Nutrição Social e Aplicada do Instituto de N utrição Josué de Castro da U niversidade Federal do Rio de Janeiro. Rio de Janeiro, RJ, Brasil 


\section{Influência do viés de seleção e de aferição em estimativas de tendência secular da estatura baseadas em dados da Marinha do Brasil*}

\section{The influence of selection and measurement bias on secular trend in height estimates from Brazilian Navy data}

\section{Gilberto Kac}

Departamento de Nutrição Social e Aplicada do Instituto de Nutrição Josué de Castro da Universidade Federal do Rio de Janeiro. Rio de Janeiro, RJ, Brasil

\section{Descritores}

Estatura, tendência secular\#. Militares". Antropometria, história\#. Viés (epidemiologia)". Fatores socioeconômicos. Nível de saúde. Brasil.

Keywords

Body height". Military

personnel. Anthropometry,

history". Bias (epidemiology).

Brazil.

\section{Resumo}

São apresentados dados sobre a ocorrência de viés nas estimativas de tendência secular em estatura, baseadas em dados da Marinha do Brasil. Foram analisados três bancos de dados; um para o período entre 1940 e 1965 e dois entre 1970 e 1977.

\begin{abstract}
Data of bias occurrence of secular height trend estimates based on Brazilian Navy data are presented. Three data sets were analyzed; one for the 1940-65 period and two for the 70 s.
\end{abstract}

\section{INTRODUÇÃO}

São escassos os estudos enfocando o processo de tendência secular em estatura (TSE) no Brasil, seja pela ausência de tradição de investigações sobre o tema, seja pela escassa disponibilidade de dados para análise.

Tradicionalmente, dados de estatura aferidos em instituições militares têm sido utilizados para estimar a TSE em diversos países da Europa e nos Estados Unidos. ${ }^{4}$ No Brasil, recentemente, Kac e Santos ${ }^{2}$ avaliaram a TSE em recrutas e alistados da Marinha do Brasil, nascidos entre 1970 e 1977, e observaram aumentos na estatura média de 0,315 e $0,221 \mathrm{~cm} /$ ano, respectivamente.
A presente nota mostra dados sobre a ocorrência de viés de aferição nas medidas de estatura que originam as estimativas de TSE baseadas em registros da Marinha do Brasil. Também são feitas considerações metodológicas sobre a utilização de dados de estatura provenientes de instituições militares para a caracterização de TSE.

\section{MÉTODOS}

A análise foi conduzida com base em três bancos de dados com as seguintes variáveis: estatura em centímetros, escolaridade, data e local de nascimento e data da aferição. Para o período entre 1940 e 1965, os dados foram coletados a partir de um arquivo localizado na

* Baseado na dissertação de Mestrado apresentada à Escola Nacional de Saúde Pública da Fundação Oswaldo Cruz, 1996. Apresentado no "The Eight International Congress of Auxology Human Growth and Development", Philadelphia, Pennsylvania, 1997.

Recebido em 18/5/1999. Aprovado em 14/9/1999 
Diretoria de Pessoal Militar da Marinha do Brasil (DPMM), situada na cidade do Rio de Janeiro. Após diversos critérios de exclusão, a amostra constituiu-se de 3.269 recrutas. As análises foram realizadas segundo os seguintes qüinqüênios de ano de nascimento: 1940-44, 1945-49, 1950-54, 1955-59 e 1960-65. Para o período entre 1970 e 1977, dois bancos de dados foram disponibilizados pela DPMM; um para alistados e outro para recrutados. A amostra final constituiu-se de 52.574 alistados, nascidos entre 1970 e 1977, e 4.459 recrutados, nascidos entre 1971 e 1976.

As análises sobre a presença de viés de aferição foram realizadas com base na distribuição de freqüência do último dígito de estatura. Inicialmente foi realizado um teste de aderência à distribuição uniforme com o objetivo de identificar a ocorrência de concentração em algum dígito. Em seguida, testou-se, através do qui-quadrado (comparação entre proporções), se essas proporções mantiveram-se constantes ao longo das séries.

\section{RESULTADOS}

A Figura apresenta as freqüências para o último dígito dos valores de estatura de recrutas da Marinha do Brasil nascidos entre 1940 e 1965. Verificou-se para todos os qüinqüênios do período uma concentração nos valores de estatura terminados em 0 e $5\left(x^{2}=38,8\right.$; g.l. $=9, \mathrm{P}=0,000012)$. Análises comparativas entre as proporções de cada dígito final de estatura ao longo dos qüinqüênios não revelaram diferenças estatisticamente significativas.

A distribuição de frequiência do último dígito de estatura para alistados e recrutados nascidos na década de 70 (resultados não mostrados) também revelaram concentração nos dígitos 0 e 5 . O valor do quiquadrado foi de $145,8(\mathrm{~g} .1 .=63, \mathrm{P}=0,001)$ para alistados e 70,8 (g.l. $=45, \mathrm{P}=0,01$ ) para recrutados. As análises temporais, a exemplo do observado para o perío-

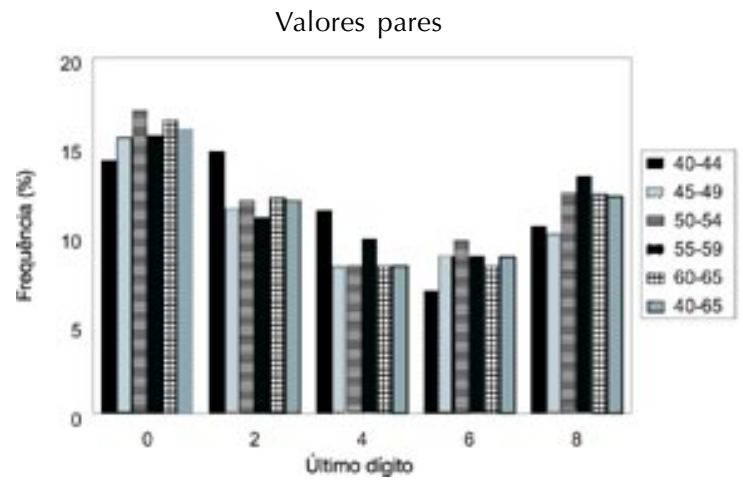

do 1940/65, também não revelaram diferenças estatisticamente significativas ao longo da série, seja para recrutados seja para alistados.

\section{DISCUSSÃO}

Existe muita discussão acerca da utilização de dados de estatura provenientes de organismos militares para estimar TSE, principalmente em relação à existência de viés. ${ }^{1,3}$ Entre estes destacam-se o viés de seleção e o de aferição.

O primeiro tipo denomina-se viés de auto-seleção de voluntários e a questão que se coloca é a seguinte: até que ponto os indivíduos selecionados são representativos das populações a que pertencem? Fogel et al ${ }^{1}$ consideram como certa a existência desse tipo de viés. A abordagem para esse problema segundo esses autores é proceder a uma comparação entre as características dos voluntários em épocas de paz, com indivíduos do mesmo subgrupo aptos ao recrutamento em períodos de guerra. Análises com base nos registros de estatura, tomados durante a Guerra Civil dos Estados Unidos, mostraram que voluntários selecionados de um subgrupo particular eram representativos dos subgrupos a partir dos quais foram selecionados. ${ }^{1}$

No Brasil não há como abordar diretamente esta questão; primeiro porque o serviço militar é obrigatório para todos os indivíduos com 18 anos, não existindo a figura do voluntário; e segundo porque não estão disponíveis registros de estatura para o conjunto de alistados para o período da Segunda Guerra Mundial.

Um outro ponto, que também merece atenção, é o seguinte: será que os indivíduos rejeitados pelas Forças Armadas por outros fatores que não a estatura são mais baixos que os incorporados? Os dados analisados por Karpinos ${ }^{3}$ para a Segunda Guerra Mundial, para o Exército do Estados Unidos, referem que $41 \%$ dos alistados foram rejeitados e que estes foram em média 0,56

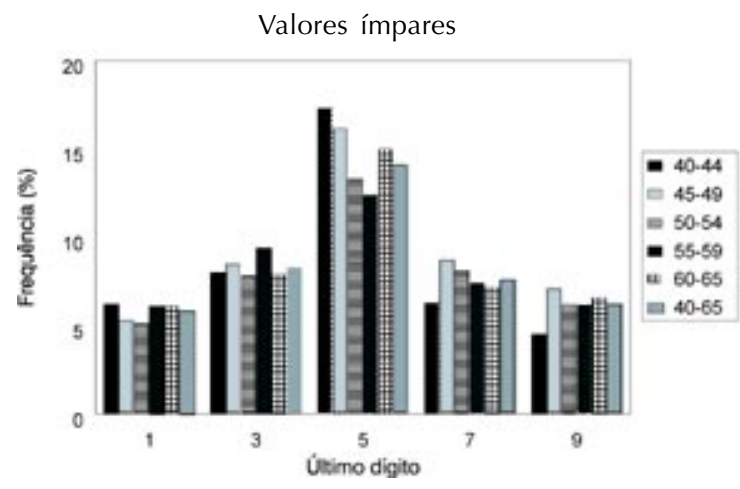

Figura - Freqüência do último dígito de estatura para recrutados pela Marinha do Brasil, segundo qüinqüênio de nascimento. Brasil, 1940 a 1965. 
$\mathrm{cm}$ mais baixos do que os recrutados. Isso significa uma diferença de $0,23 \mathrm{~cm}$, o que somente em grandes amostras torna-se estatisticamente significativo.

Os dados analisados por Kac e Santos, ${ }^{2}$ por contemplarem informações tanto para alistados como para recrutados, apontaram para questões bastante peculiares no que diz respeito ao processo de seleção de recrutas da Marinha do Brasil. Curiosamente, ao contrário do que se pode imaginar, a Marinha brasileira não recruta necessariamente indivíduos com maior estatura ou escolaridade. Quanto à estatura, alistados apresentaram médias mais elevadas do que recrutados para a maioria das macrorregiões do País no período 1971/76. No que diz respeito à escolaridade, os autores evidenciaram que a Marinha preferencialmente seleciona indivíduos com exatamente 8 anos de estudo. ${ }^{2}$ Dessa forma, percebe-se que os critérios que orientam a seleção de recrutas da Marinha do Brasil diferem do padrão reportado pela literatura internacional, que é o de recrutar sempre os indivíduos mais altos. Sendo assim, as estimativas de TSE baseadas em dados da Marinha do Brasil, não devem ser diretamente extrapoladas para a população brasileira em geral, já que tanto alistados como recrutados apresentam um perfil antropométrico e socioeconômico diferente da população em geral. ${ }^{2}$

$\mathrm{O}$ viés de aferição pode comprometer tanto a idade como a estatura. Em relação à estatura, a preocupação reside nos arredondamentos ou ainda segundo o grau de precisão. Os arredondamentos, geralmente para valores pares, ocorrem mesmo em situações onde a estatura é aferida por pessoal qualificado, como foi o caso do inquérito nacional conduzido entre 1960/62 nos Estados Unidos. Modelos de simulação, entretanto, não indicaram introdução de viés nessas situações. ${ }^{1}$ Apesar de exercer um efeito na acurácia das estimativas de estatura média, esse erro não ultrapassou $0,24 \mathrm{~cm}$, nos casos em que ocorreram de $15 \%$ a $30 \%$ de arredondamentos. Segundo análises de dados da Segunda Guerra Mundial, ${ }^{3}$ a tendência atual é de se arredondar o valor da estatura para baixo, o que não distorce análises de TSE por não introduzir erros de magnitude relevante.

No presente estudo, verificou-se uma importante concentração do último dígito de estatura nos valores terminados em 0 e 5, seja para o período entre 1940/65, seja para os dados contemporâneos (1970/77). Os resultados dessas análises sugerem, no entanto, que mesmo estando presente, o viés de aferição distribuiuse de forma relativamente homogênea nos três bancos de dados analisados ao longo das séries temporais, indicando que as estimativas sobre TSE não foram distorcidas por este tipo de viés.

Em resumo, os resultados do presente estudo mostraram que apesar da reconhecida existência de viés de aferição nas estimativas de TSE, baseadas nos dados de estatura da Marinha do Brasil, essa fonte de dados constitui-se em uma importante alternativa para avaliar a TSE, principalmente na ausência sistemática de inquéritos antropométricos de representatividade nacional.

\section{REFERÊNCIAS}

1. Fogel RW, Engerman SL, Floud R, Friedman G, Margo RA, Sokoloff KL et al. Secular changes in American and British stature and nutrition. I Interdiscip Hist 1983;15:445-81.

2. Kac G, Santos RV. Secular trend in height in enlisted men and recruits from the Brazilian Navy born from 1970 to 1977. Cad Saúde Pública 1997;13:479-87.

3. Karpinos BD. Height and weight of selective service registrants processed for military service during world war II. Hum Biol 1958;30:292-321.

4. Van Wierigen JC. Secular growth changes. In: Falkner F, Tanner JM, editors. Human growth: a comprehensive treatise. New York: Plenum Press; 1986. p. 307-31. 